\title{
EDITORIAL
}

\section{Righting scientific wrongs}

\author{
The response from the scientific community to two recent controversies has shown that \\ although scientific progress is not always linear, the right answer will emerge in the end.
}

Science in its most ideal form is the search for an understanding of nature or, more philosophically, a search for the truth. Science moves forwards through a conversation between researchers: a question is experimentally addressed, the results are communicated and published, and then further experiments are carried out in different laboratories. Initial findings are often met with scepticism, but when further experimentation corroborates these findings, they are generally widely accepted as fact and become part of the collective knowledge. However, subsequent experimentation can also show that the initial findings were incorrect. For example, in 1953 Pauling and Corey reported that DNA forms a triple helix with the phosphate groups located at the core ${ }^{1}$. However, soon after, Watson and Crick showed that DNA in fact forms a double helix with the phosphates on the outside ${ }^{2}$, a finding that has since been corroborated many times. Two recent examples have highlighted that this process of experimentation-publication-retesting is a viable, albeit circuitous, mechanism that allows us to ensure that our collective scientific knowledge is as factually accurate as possible.

At the end of 2010, Wolfe-Simon et al. reported that a bacterium of the genus Halomonas can substitute arsenic for phosphorus to sustain its growth, incorporating arsenate into macromolecules that normally contain phosphate, such as $\mathrm{DNA}^{3}$. This finding received widespread media attention but was met with scepticism in the scientific community, as such substitutions for any of the major elements that are essential for life had not been reported before. Indeed, soon after the paper was published, much comment and discussion ensued online, questioning not just the findings themselves but also the nature of the publication process and media courting. A journal has much to gain by publishing articles that garner such attention, but must also be responsible for any potential follow-up. Science, which published the original report, has now published eight thoughtful letters that reflect this online debate, raising doubts about the original findings, as well as a response from the authors. This debate remains to be settled, but the ongoing conversation between the researchers will move us closer to the correct answer and may reveal interesting new biology in the process.

In the second example, in October 2009, Lombardi et al. proposed a causative link between xenotropic murine leukemia virus-related virus (XMRV) and chronic fatigue syndrome $(\mathrm{CFS})^{4}$. Since then, at least ten studies have been unable to confirm the proposed link, and the initial detection of the virus is now widely considered to be the result of contamination. In this case, the experimentation-publication-retesting process has clearly worked; the response from the scientific community has all but discredited the initial erroneous link between XMRV and CFS.

However, science does not operate in a vacuum, and its results may have direct clinical implications. For example, the American Red Cross responded to the XMRV story by calling for a ban on blood donations from persons with CFS, thereby missing out on life-saving donations, and some people with CFS have started expensive antiviral treatments in search of a cure. An example of an even more harmful controversial finding is the now thoroughly discredited report that the Measles-Mumps-Rubella vaccine can lead to autism ${ }^{5}$; this report led directly to a decrease in the UK vaccination rate and higher disease rates. Because of such potential to needlessly scare the public or induce premature responses, scientists and the media have a duty to be aware of the potential outcomes of their work and should not be tempted to make sweeping claims. The possibility always remains that the result was incorrect owing to circumstances outside the researchers' control. So, it is alright to be wrong - but we should always be very cautious when reporting controversial findings.

As these examples show, the process of experimentation-publication-retesting is a powerful mechanism that has withstood the test of time. And now that the speed of communication, through various online forums, has accelerated to become almost instantaneous, and faster and more powerful tools for research become available almost daily, new findings can be evaluated even faster by the community. The scientific record will hopefully therefore become more and more accurate.

\footnotetext{
. Pauling, L. \& Corey, R. B. A proposed structure for the nucleic acids. Proc. Natl Acad. Sci. USA 39, 84-97 (1953).

2. Watson, J. D. \& Crick, F. H. C. Molecular structure of nucleic acids: a structure for deoxyribose nucleic acid. Nature 171, 737-738 (1953).

Wolfe-Simon, F. et al. A bacterium that can grow by using arsenic instead of phosphorus. Science 332, 1163-1166 (2011).

4. Lombardi, V. C. et al. Detection of an infectious retrovirus, XMRV, in blood cells of patients with chronic fatigue syndrome. Science 326 , 585-589 (2009).

5. Wakefield, A. J. et al. Ileal-lymphoid-nodular hyperplasia, non-specific colitis, and pervasive developmental disorder in children. Lancet 351 637-41
} 